\title{
VIII. NATION UND GESCHLECHT
}

$\mathrm{Zu}$ den zeitgenössischen Definitionsmerkmalen der Nationsidee im Kontext öffentlicher Denkmaleinweihungen in den Hauptstädten gehörten im Betrachtungszeitraum neben vergleichsweise rigiden politischen und religiösen auch geschlechtsspezifische Kriterien. Frauen tauchten seltener in den Reihen der Gäste auf, sie hielten keine Festreden und waren in der Regel höchstens als Begleitung ihrer Männer erwünscht. Sowohl in London als auch in Berlin blieb die emanzipatorische Freigabe der Denkmalsockel für Frauen auf Dynastinnen beschränkt und wahrte insofern einen dezidiert elitären Charakter der Ehrung. DaB entsprechende Würdigungen zustandekamen, nahm sich für London weniger einschneidend aus, sofern hier bereits die überlange Regentschaft der Monarchin dazu zwang, die Nation mit einer weiblichen Kultfigur in Verbindung zu bringen. In Paris schließlich ist die monumentale Reminiszenz an weibliches Personal zwar gelungen, zeugte aber eine noch größere Zahl gescheiterter Projekte von dem Umstand, daß eine entsprechende Deutungsmacht keineswegs als gesichert gelten konnte.

\section{Paris: Gescheiterte Projekte}

Um 1907 unternahm ein Komitee den Versuch einer Denkmalerrichtung für die Autorin und anarchistische Sozialistin Louise Michel. Der Subskriptionsaufruf richtete sich ausdrücklich an die "travailleurs«, deren zahlreiche Mitwirkung im Sinne einer »souscription essentiellement populaire« erwünscht war $^{1}$. Die Statue, deren Modell von Émile Derré bereits als Photo vorlag, sollte demnach dokumentieren "que nous ne trouverons ni dans le mensonge des religions, ni dans la morale de notre société corrompue, notre idéal de beauté et de justice, mais dans l'amour et la bonté, dont Louise fut l'incarnation ${ }^{2}$. Intendiert war neben dem politischen Bekenntnis zu humanitären Werten vor dem Hintergrund deutlicher Autoritäts-, Religions- und Gesellschaftskritik ein regelrechtes Gegendenkmal, eine »belle figure du Peuple« und »hommage du Prolétariat «, die den engen Rahmen der üblichen Initiatoren- und Subskribentenkreise zugunsten der Arbeiterklasse sprengen sollte ${ }^{3}$. Im Juni des Jahres

1 Comité de souscription pour le Monument Louise Michel [Subskriptionsaufruf], AP VM 92/1 Dossier: Monument à Louise Michel.

Ibid.

Vgl. ibid. 
wandte sich eine Gruppe, der u.a. der Kopf der republikanischen Ligue des droits de l'homme, Francis de Pressensé, der seit Mitte der 1880er Jahre im Conseil von Paris amtierende Radikalsozialist Édouard Vaillant und der sozialistische Possibilist Paul Brousse angehörten, in einer Depesche an den Präsidenten des Conseil Municipal und erbaten die Aufstellung der Statue an öffentlichem Ort in Montmartre. Sie rechtfertigten die Platzwahl mit dem Argument, daß die Place des Abbesses mit ihren »dimensions modestes, son caractère d'intimité familiale«, besonders geeignet erscheinen mußte. Damit vermieden die Antragsteller offensiv linksradikale Voten und kaschierten ihr politisches Credo hinter der neutralisierenden Idee besonderer "abnégation « und "courage« der Louise Michel, mit der die Monumentalsymbolik nun eingefordert wurde ${ }^{4}$.

Zwar leitete der Conseil Municipal die Bitte an die Administration weiter', der Vorstoß blieb aber erfolglos. Zwei Jahre später lag noch einmal ein Schreiben des Komitees vor, das wiederum verklausulierend den "caractère essentiellement populaire « des Denkmals betonte und neue Standorte im (symbol)politisch republikanischen Osten der Stadt vorschlug, an denen die Denkmalerrichtung doch noch möglich werden sollte ${ }^{6}$. Damit verloren sich aber bereits die Spuren des Projekts, das sich für die radikale Linke als nicht durchsetzungsfähig erwies. Im Projektstadium stagnierte ebenfalls ein Denkmal für die Literatin Mme de Staël, für das sich ein ausschließlich aus adligen »femmes de lettres« gebildetes Komitee einsetzte ${ }^{7}$. Der Conseil Municipal billigte noch die angefragte Subvention für das bereits vorliegende Modell ${ }^{8}$, eine Aufstellung unterblieb jedoch, ohne daß sich abgezeichnet hätte, woran das Projekt scheiterte.

Auch 1909 angestellte Überlegungen zu einem Denkmal, das eigens die Einbeziehung der Frauen in den Niederlagenmythos der Nation leisten sollte, indem es als obeliskförmig-abstraktes »Monument de la Femme française« das kollektive Verdienst der Frauen um die stolze, wenn auch erfolglose Widersetzung gegen den deutschen Feind 1870/71 im von den Angriffen besonders drangsalierten Montrouge ganz im Süden der Kapitale verewigen sollte, blie-

e Dixneuvième Siècle, 12 Juni 1907, AP ibid

Vgl. Rapport du Conducteur Wallers vom 19. August 1907, ibid.

6 Vgl. Brief des Komitees vom 6. Juni 1909, ibid. Vorgeschlagen wurden Quai Valmy et Jemmapes im 10. Arrondissement nahe der Place de la République, noch weiter östlich die Place des Armandiens im 20. Arrondissement und die rue d'Angulème östlich der Place de la République im 11. Arrondissement.

7 Vgl. Protokoll des Inspecteur en Chef des Beaux-Arts vom 10. März 1909, DAC Dossier: Mme de Staël. Das Komitee plädierte für eine zentrale Aufstellung am Boulevard Malesherbes am Übergang vom 1. zum 8. Arrondissement. Vgl. Brief der Mme d'Uzès an den Préfet de la Seine vom 1. März 1909 und Protokoll der Direction des Affaires des services d'architectures vom 9. Juli 1909, ibid.

8 Vgl. Protokoll der Conseil-Sitzung vom 7. April 1909, ibid. 
ben Episode ${ }^{9}$. Inschriftlich hatte hier auch die Übernahme des Märtyrer- und Heldentopos für die Frauen angestanden, die als »héroïnes de 1870/71« für "courage - dévouement - résignation « geehrt und damit symbolisch zum integrativen Bestandteil einer "page consolante de notre histoire« und also ebenso einer heroisierten wie emotionalisierten Geschichtserinnerung aufrücken soll$\operatorname{ten}^{10}$.

Gescheitert ist schließlich auch das Projekt für ein Denkmal, mit dem an die Philosophin und Anthropologin Clémence Royer erinnert werden sollte, die in Frankreich vor allem als Übersetzerin Darwins und Teilnehmerin der Debatte zwischen katholischer Kirche und Antiklerikalen um die Evolutionstheorie bekannt geworden war $^{11}$. Daß in diesem Falle die ungünstigen Bedingungen im unmittelbaren Vorfeld des Krieges ausschlaggebend waren und der Umstand, daß die Monumentalisierung einer politisch konnotierten Frauenfigur nicht leicht durchsetzbar gewesen wäre, legt bereits das prominente Profil der Denkmalinitiatoren nahe. Zum vom Nachlaßverwalter Royers und Fabrikanten Albert Colas ins Leben gerufenen Komitee gehörten nämlich neben dem ehemaligen Ministerpräsidenten Georges Clemenceau der mehrmalige Ministerpräsident und Außenminister Aristide Briand, Arbeitsminister René Viviani, Émile Levasseur vom Institut de France und Anatole France von der Académie Française sowie zahlreiche Vertreterinnen von Frauenvereinen wie die Generalsekretärin des Nationalrats der Femmes françaises, die Ehrenpräsidentin des Conseil national des Femmes de Saint-Lazare, die Präsidentin der Ligue française pour les Droits de Femmes und die Vorsitzende der Union de pensée féminine $^{12}$. Damit standen nicht nur tendenziell linksradikale und organisierte Fraueninteressen, sondern auch hochrangige Vertreter amtierender oder ehemaliger Regierungen in großer Zahl hinter dem Projekt.

Auf Bitten des Komitees erwarb der Staat das Gipsmodell einer RoyerFigur, das bereits 1912 öffentlich ausgestellt worden war. Um die Bronzeversion, die dann wohl auf Kosten des Komitees inzwischen hergestellt worden war, vor kriegsbedingter Einschmelzung zu schützen, wandte sich das Komitee

9 Vgl. Schreiben des Präsidenten eines Komitees vom 23. April 1909, AN F ${ }^{21} 4855$ Dossier: Monument de la Femme Française de 1870-1871, par E. Boverie, sculpteur, et Charles Ferrant, architecte, 20 avril- 22 juillet 1909 [up], mit Photo.

10 Vgl. ibid. und Notiz des Architecte des promenades vom 7. Dezember 1909, AP VM 92/1 [up].

11 Vgl. CARON, Frankreich im Zeitalter des Imperialismus, S. 122.

12 Vgl. Comité du monument Clémence Royer [vermutlich Teil eines Subskriptionsaufrufs] AN F ${ }^{21} 4856$ Dossier: Monument Clémence Royer, statue du sculpteur Henri Godet, 15 septembre 1907-12 mars 1928. An der Initiative beteiligten sich darüber hinaus u.a. der ehemalige Industrieminister und Sozialist Mesureur, 11 Deputierte, drei Senatoren, vier Mitglieder des Conseil Municipal de Paris, 19 Schriftsteller und Publizisten, darunter der Chefredakteur des Le Radical Henry Maret, acht Schriftstellerinnen, ein Komponist und ein Bildhauer, zwei Lehrer, vier Professoren, der Vorsitzende einer Tuberkulosestiftung und der Präsident der Libre Pensée in Genf. 
noch im Februar 1918 an den Staat, den es um den Erwerb und die Magazinierung der Bronze bat, die nach dem Krieg "sur une place publique en France» ausgestellt werden sollte ${ }^{13}$. Über eine öffentliche Aufstellung wurde weiter nachgedacht ${ }^{14}$. Eine Kommission der Beaux-Arts kam allerdings im Rahmen eines Gutachtens zu dem Schluß, daß dem Bronzedenkmal der Clémence Royer keinerlei »valeur décorative« zukomme, so daß von einer öffentlichen Aufstellung Abstand zu nehmen sei ${ }^{15}$. Das Verfahren endete zunächst mit dem Votum eines beauftragten Architekten, der die Plazierung lapidar am ehesten in einer »cité ouvrière« für geraten hielt, ohne sich auf Begründungen einzulassen. Ganze zehn Jahre später tauchten erneut Spuren eines Errichtungsplans auf, denen zufolge die Aufstellung im Jardin du Luxembourg oder im Jardin des Plantes erwogen wurde ${ }^{16}$, führten jedoch zu keinem Ergebnis. Pläne zur emanzipatorischen Würdigung von Frauen zeichneten sich damit für Paris zwar in mehreren Fällen ab, ausbleibende Einweihungsfeiern verhinderten aber, daß programmatische Aufladungen und nationale Konnotierungen öffentlichkeitswirksam entfaltet werden konnten ${ }^{17}$.

\section{Berlin: Dynastische Repräsentationsgrenzen}

In Berlin konnte die Denkmalwürdigung von Frauen nur glücken, weil sie in den engen Grenzen des dynastischen Repräsentationsvorbehalts blieb. Am 10. März 1880 erhielt Königin Luise nach dem etwas marginalisierten Standbild in Oranienburg von 1858 ein weiteres Denkmal im Zentrum der Luiseninsel im Tiergarten ${ }^{18}$. Auf Anordnung des Kaisers sollte der Tag als »Familienfest« begangen und daher übermäßiger »königlicher Prunk« vermieden wer-

13 Vgl. Notiz vom 12. Februar 1918, AN ibid.

14 Vgl. Bericht des Architecte des Promenades vom 12. Mai 1919 und Bericht desselben vom 4. Juni 1921, AP VM 92/1 Dossier Monument à Clémence Royer, Square de la Sorbonne.

15 Vgl. Mitteilung an den Directeur général des travaux vom 8. Dezember 1921, AP ibid.

16 Vgl. Mitteilung des Directeur de l'Enseignement supérieur an den Directeur des BeauxArts vom 12. März 1928, AN ibid.

17 Zustandegekommen ist statt dessen durchaus ein Denkmal für die emanzipierte Schriftstellerin George Sand, das aber weniger an die frühsozialistische Überzeugung der Autorin erinnerte, sondern sich im Jardin du Luxembourg ganz auf die Ästhetisierung der Figur verlagerte. Vgl. zu dem am 1. Juli 1904 aufgestellten Denkmal HARGROVE, Les statues de Paris, La représentation, S. 246. 1914 erhielten die beiden Philantropinnen Marguerite Boucicault und Clara de Hirsch ein Doppeldenkmal im 7. Arrondissement. Vgl. Érection, square des Ménages, d'un monument à Mme Boucicaut et la Baronne Hirsch, séance du 14 juin 1911, in: CMPV, Année 1911, $1^{\text {ire }}$ partie, S. 993-994.

18 VZ, 10. März 1880 (A.), S. 2. 
$\operatorname{den}^{19}$. Entgegen sonstiger ritueller Gepflogenheit brachte die Anwesenheit des Kaisers bei der Denkmaleinweihung hier nun keine Militarisierung der Festszene mit sich, indem der Monarch zwar in Uniform erschien, ansonsten aber nur Choräle und die Hymne ertönten.

Die Vossische Zeitung publizierte am Festtag einen kurzen Artikel, indem sie den Akt quasi-religiös deutete und die "Wallfahrt" der "Nachkommen« Luises zum Festplatz als Sinnbild beschwor: „Die Kaiser-Mutter tritt in unverwüstlicher Gestalt in das Volk zurück (...), sie feiert ein Auferstehen im Glanz der nationalen Größe und lebt mit den Nachkommen, wie sie mit den Vorfahren gelebt hat $\kappa^{20}$. Im Deutungsmuster dominierte damit neben der bereits für das Oranienburger Denkmal kennzeichnenden metaphysischen Auslegung der Denkmalerrichtung als Epiphanie, die Zuordnung der Luise vor allem zum »Volk«, dessen Vision von »nationaler Größe« in ihr die populäre Repräsentantin fand ${ }^{21}$. Daneben diente ihre Identifizierung als Mutter des Kaisers einer genealogischen Kontinuitätsstiftung zwischen der politischen Reichsgegenwart und dem Preußen des frühen Jahrhunderts.

Vor allem aber veröffentlichte die Vossische einen Brief Luises, der an der biographischen Schnittstelle der inzwischen längst traditionellen Mythosbildung stand, indem er sich auf die private Begegnung Luises mit Napoleon in Tilsit im Juli 1807 bezog $^{22}$. Daß sie ungeachtet ihres patriotischen Stolzes und notorischen Napoleon->Hasses mit ihrem Volk leidend den Opfergang antrat, um günstige Friedensbedingungen für ihr Land zu erbitten, hatte sie dem Mythos zufolge zur Märtyrerin gemacht. Daß sie sich im veröffentlichten Brief nun zu ihrer Feindseligkeit gegen Napoleon und zur Bitterkeit über die ihr abverlangte Geste bekannte, die sie gleichwohl »mit der Würde der Preußischen Königin« zu ertragen bereit war, sollte das längst etablierte Deutungsmuster bekräftigen.

Das auf diesem Wege entworfene Idealbild schwankte zugleich zwischen dieser Stilisierung zur »Auserwählten« und dem eher bürgerlichen Werthaltungen folgenden Lob als "Muster der reinsten Weiblichkeit«, das sie als »Frau, Mutter und Gattin« abgegeben habe ${ }^{23}$. Während sie der Missions- und Opfermythos entrückte, band sie der bürgerliche und spezifisch weibliche Sittenkodex wieder näher an das "Volk«, mit dem sie über Verhaltensnormen einschließlich ihrer »königlichen Pflicht für den Staat«, vor allem aber über ih-

$19 \mathrm{Zu}$ den Ehrengästen zählten neben Fürsten und Fürstinnen Generale, Deputationen von Magistrat und Stadtverordneten sowie Vertreter der Friedrich-Wilhelm-Universität.

$20 \mathrm{VZ}, 10$. März 1880 (M.), S. 1.

21 Vgl. Regina SCHULTE, Der Aufstieg der konstitutionellen Monarchie und das Gedächtnis der Königin, in: HA 6 (1998) S. 76-103, hier S. 91.

22 Vgl. VZ, 10. März 1880 (M.), S. 1.

23 Vgl. ibid. 
re Empathie und ihren »Dank für die Opfer des Volks« eng verbunden gedacht werden konnte ${ }^{24}$.

Damit nahm sie sich am Ende bei aller mystischen Überhöhung als eine spezifisch nahbare, emotionsbesetzte Kultfigur aus, über die man an die Nation zunächst als historische Leidens- und nach den Befreiungskriegen als Triumphgemeinschaft anknüpfen konnte. Neben der "geschichtlichen Größe der Königin« blieb der »Zauber der Frau«, neben der historisch-politischen die weiblich-moralische Qualität die eigentliche Quintessenz ihrer Exempelfunktion und das Spezifikum einer Figur, die sich mehr und anders als jedes männliche Pendant dazu zu eignen schien, der feiernden Nation nicht nur bewunderndes Erinnern abzuverlagen, sondern auch gleichsam ein affektives Identifikationsangebot zu unterbreiten. Die antinapoleonische Wendung und also ein dem französischen durchaus vergleichbarer Niederlagentopos war zugleich konstitutiver Bestandteil des Luisenkults, denn die Retterfigur brillierte einmal mehr als große Antagonistin des brutalen Bezwingers Preußens.

Der Kommentar der Neuen Preußischen Kreuz-Zeitung wich von diesen Sinnzuschreibungen nicht wesentlich ab, legte aber zugleich größeren Wert darauf, das Luisendenkmal konstitutiv in die bereits etablierte Denkmallandschaft einzubeziehen und brachte es daher gleichermaßen mit der ViktoriaAllegorie auf dem Brandenburger Tor und dem »landesväterlichen Königsbild« Friedrich Wilhelms III. im Tiergarten als Symbolen triumphaler Preußenmacht und eines seinerseits nahbar-bürgerlich gedeuteten Königtums in Verbindung ${ }^{25}$.

Die affektive Komponente trat hinter machtpolitisch-historischen Assoziationen zurück, indem als Quintessenz des Luisenkults die Stiftung des Eisernen Kreuzes am Geburtstag der Königin durch Friedrich Wilhelm III. erschien, die Wilhelm I. im Juli 1870 wiederaufgegriffen hatte, um diejenigen auszuzeichnen, die sich wie die mittelalterlichen Ritter im "Kampfe gegen Unchristen und Undeutsche« als dem Inbegriff des Feindbildes der Nation bewährt hatten. Die Assoziation des karitativen Luisenordens und der Tätigkeit seiner weiblichen Mitglieder im Sanitätsdienst während der Befreiungskriege und im Krieg 1870/71, die neben der Stiftung des Eisernen Kreuzes die zweite zentrale, auf die Kultfigur gegründete Traditionsstiftung darstellte, wurde von der Kreuz-Zeitung gemieden, und somit die militärische gegenüber der emanzipatorischen Traditionsbildung deutlich favorisiert. Dies, obschon im Bildprogramm des Denkmalsockels gerade diese Sonderrolle Luises ausdrücklich thematisiert wurde ${ }^{26}$ und ein entsprechender Verweis auf die Ikonographie

24 Vgl. ibid.

${ }^{25}$ Vgl. NPKZ, 11. März 1880, S. 1.

${ }^{26}$ Die Relieffolge entlang des Rundsockels, auf dem die Luisenfigur stand, zeigte Szenen aus den Freiheitskriegen, darunter als »Bild der Frauenthätigkeit« im Krieg die Pflege der Verwundeten und spielte damit auch auf die historische Rolle des Luisenordens als Beförderer 
hätte naheliegen können. Umgekehrt erwies sich die Kreuz-Zeitung durchaus als Chronist der politischen Rolle Luises, indem sie an die Rückberufung Hardenbergs durch den preußischen Regenten und an den refombedingten Leistungsschub des allmählich regenerierten Preußen erinnerte und dafür Luise mit verantwortlich machte ${ }^{27}$.

In Reden und Kommentaren zum Denkmal wurde somit die weibliche Qualität der Figur nicht im Hinblick auf die Inklusion von Frauen im Rahmen des Luisenordens gedeutet. Sie diente eher dazu, bürgerliche Funktionszuschreibungen und Moralkodizes im Hinblick auf die "Frau, Gattin und Mutter« abzurufen. Damit sind in der Tat nur im Rahmen einer umfassenden zeitgenössischen Vortrags- und Aufsatz-Literatur längst kanonisierte Deutungsmuster wiederaufbereitet worden. Bereits im Rahmen der Feiern zum 100. Geburtstag der Königin im März 1876 hatte nämlich Heinrich von Treitschke seine Festrede dazu genutzt, selbst der gefeierten Repräsentantin ihres Geschlechts politisch aktive Teilhabe an der Nation unwiderruflich zu bestreiten:

Wie die Reformation unserer Kirche das Werk von Männern war, so hat auch dieser preußische Staat (...) allezeit einen bis zur Herbheit männlichen Charakter beansprucht. (...) Keine aus der langen Reihe begabter Fürstinnen (...) hat unseren Staat regiert. Auch Königin Luise bestätigt nur die Regel. Ihr Bild (...) ward eine Macht (...), doch nie mit einem Schritte übertrat sie die Schranken, welche der alte deutsche Brauch ihrem Geschlechte setzt ${ }^{28}$.

Demgegenüber veröffentlichten in der Flut der Luisenliteratur nach der Jahrhundertwende die liberalen Grenzboten einen Artikel, der Treitschkes Deutungsmuster unterlief und Mythenkritik betrieb. Die Vita der Königin wurde nun nach den "Beweisen starker Selbstbethätigung auf politischem Gebiete $\aleph^{29}$ abgesucht und die Überwindung anfänglicher »unheilvolle[r] Passivität« gerade zu einer Art Peripetie innerhalb einer fortan primär politischen Biographie gedeutet. Luise changierte so zum Prototyp der $»$ Germanenfrauen ${ }^{30}$ und erhielt, was Treitschke ihr im Namen des Mythos verweigert hatte: »einen Platz in der ersten Reihe derer (...), die den preußischen Staat haben bauen hel-

der Wehrhaftigkeit Preußens an. Vgl. Germania, 10. März 1880, S. 3; [A. TRENDELENBURG,] Ansprache (über die Denkmäler der Königin Luise und der Freiheitskriege in Berlin), Berlin 1914 (B. zum Jahresbericht des Friedrichs-Gymnasiums. Ostern 1914. Die Feier des 10. März) S. 11-13; MƯLLER-BOHN, Die Denkmäler, S. 42f. Vgl. zum Sockelprogramm Jutta VON SIMSON, Das Denkmal der Königin Luise in Berlin. Ein Beitrag zur Luisenverehrung im 19. Jahrhundert, in: L. GRIESEBACH, K. RENGER (Hg.), FS für O.S. zum 65. Geburtstag, Berlin 1977, S. 516-530, hier S. 524-525; SIMON, Die Bildpolitik, S. 255-256.

27 Vgl. NPKZ, 11. März 1880, S. 1.

28 Vgl. Heinrich VON TREITSCHKE, Königin Luise. Vortrag, gehalten am 10. März 1876 im Kaisersaale des Berliner Rathauses, in: Preußische Jahrbücher 37 (1876) S. 417-429, und wieder in: DERS., Historische und politische Aufsätze, Bd. 4, Leipzig 1897, S. 310-324, hier S. 311 .

30 Ibid. S. 735. 
fen ${ }^{31}$. Demgegenüber ließen die Berliner Denkmalstifter von 1880 die politisch-emanzipatorische Funktion der Figur in der Folge einer namhaften Deutungstradition weithin außer Acht.

Luise blieb nicht die einzige weibliche Kultfigur auf Berliner Denkmalterrain: Ende Oktober 1895 wurde fünf Jahre nach ihrem Tod ein Denkmal für die Gattin Wilhelms I., Kaiserin Augusta, auf dem Opernplatz eingeweiht ${ }^{32}$. Mit der Positionierung inmitten des monarchischen Repräsentationsareals deutete sich der Versuch einer Politisierung der Gestalt bereits an und unterschied sich zugleich von der Aufstellung des Luisendenkmals, das im Tiergarten auf den zwar ebenso politisierten, zugleich aber mythisch entrückten Charakter der Kultfigur verwiesen hatte. Staatsminister, Vertreter der städtischen Behörden, Militärs, protestantische und katholische Geistliche, und hier nun erst- und letztmals in vergleichbarem Rahmen öffentlich sichtbar ein Rabbiner, verfolgten neben den Komiteemitgliedern das Festgeschehen ${ }^{33}$. Die unaufwendig kurze Zeremonie wurde am Ende aber anders als die Luisenfeier erheblich militärisch überformt, denn während Vertreter und Vertreterinnen gemeinnütziger Vereine Kränze am Denkmal niederlegten, formierte sich die Infanterie zu einem abschließenden Parademarsch, bevor der Kaiser, gezielt die TriumphstraBenarchitektur betonend, den Festplatz im Vierspänner über die Linden Richtung Brandenburger Tor verlie $B^{34}$.

Die Rede des Stadtverordnetenvorstehers Styck ordnete den neuen AugustaKult ganz in die offiziellen Feierlichkeiten zum 25-jährigen Jubiläum der Reichsgründung und in die öffentlich inszenierten Loyalitätsbeweise und Huldigungsakte gegenüber Wilhelm I., dem regierenden Monarchen, den »Heerführer[n]« und »Staatsmännern[n]« ein ${ }^{35}$. Damit stellte er die Figur zunächst den männlichen Repräsentanten der militärischen und zivilen Reichsnation gleichberechtigt zur Seite, verlegte sich dann aber ganz auf die Rolle Augustas als Organisatorin einer "freiwillige[n] Krankenpflege« an der Spitze des von ihr 1866 begründeten Vaterländischen Frauenvereins, dem sich inzwischen längst Frauenwohltätigkeitsvereine auch aus anderen deutschen Einzelstaaten angeschlossen hatten, so daß hier gleichsam die weibliche Kriegsvorbereitung professionalisiert erschien ${ }^{36}$. Ihr "großes Verdienst um das deutsche Volk«

31 Ibid. S. 740.

32 Vgl. zum Folgenden Berliner Tageblatt, 21. Oktober 1895, LAB A Rep. 000-02-01 Nr. 2381 [up], und Programm zur Feier der Enthüllung des Denkmals weiland Ihrer Majestät der Kaiserin und Königin Augusta, ibid.

${ }^{33}$ Vgl. ibid.

34 Vgl. ibid.

35 Vgl. zum Folgenden Berliner Tageblatt, 21. Oktober 1895, LAB A Rep. 000-02-01 Nr. 2381 [up]; Rede des Herm Dr. Stryck, LAB A Rep. 000-02-01 Nr. 2381 [up].

36 Vgl. Ute PlaneRT, Vater Staat und Mutter Germania: Zur Politisierung des weiblichen Geschlechts im 19. und 20. Jahrhundert, in: DIES. (Hg.), Nation, Politik und Geschlecht. Frauenbewegung und Nationalismus in der Moderne, Frankfurt, New York 2000, 
beschränkte sich der Rede zufolge gleichwohl auf die Demonstration gewissenhafter Caritas zugunsten der »erkrankten und verwundeten Krieger« sowie auf die Rolle als Erzieherin der "weiblichen Jugend « zur Krankenpflege und also gleichsam als Sekundantin der siegreichen Kriegsnation und Muster einer bürgerlichen Frauenrolle. Die Konnotation von »Volk« blieb im gleichen MaBe stark selektiv, sofern es nur entweder in Gestalt der kämpfenden Soldaten oder der in der Verwundetenpflege mit »Pflichttreue und Opferwilligkeit« arbeitenden Frauen thematisiert wurde. Jenseits der ausgemachten humanitärpädagogischen Qualifikation der Monarchin stilisierte man sie ohnedies vor allem zum Kultobjekt der »Liebe und Ehrfurcht ${ }^{37}$, so daß sie ähnlich wie Luise zuallererst als affektiver Projektionshintergrund des nationalen Kults dienen sollte ${ }^{38}$.

\section{London: Ableger des Monarchinnenkults}

Ein dynastischer Repräsentationsvorbehalt galt bis vor Kriegsausbruch auch in London für die Frauen, die zu Denkmalehre gelangten. Unscheinbar blieb die ganz dem »civic ritual« verhaftete Aufstellung der Replik einer ursprünglich bereits 1712 plazierten Queen Anne-Statue Mitte Dezember 1886 vor der St. Paul's-Kathedrale ${ }^{39}$. Die knappen Reden zum unauffälligen Akt erschöpften sich im Repetieren von Traditionsformeln, die den konservativen Bund von Staatskirche und Monarchie betrafen, oder Devotionsformeln, die sich an die regierende Monarchin wandten und also damit bereits den Victoria-Kult aus einer dezidiert hauptstädtischen Perspektive antizipierten, ohne über die institutionellen Sinnbezüge für die nationale Ordnung hinauszugelangen ${ }^{40}$. Eine Sonderfunktion der immerhin bis dahin seltenen Frauenfigur als nationaler Referenzpunkt wurde nicht thematisiert.

Präzisere Deutungsmuster wurden demgegenüber erkennbar, als im Juli 1908, während der monumentale Victoria-Kult auch in London längst begon-

S. 15-65, hier S. 39; Karen HAGEMANN, "Deutsche Heldinnen«: Patriotisch-nationales Frauenhandein in der Zeit der antinapoleonischen Kriege, in: ibid. S. 86-112; Ute FREVERT, Nation, Krieg und Geschlecht im 19. Jahrhundert, in: HETTLING, NOLTE ( $\mathrm{Hg}$.), Nation und Gesellschaft in Deutschland, 1996, S. 151-170.

Vgl. zum Folgenden The Morning Post, 6. Juni 1888, S. 7; The Times, 16. Dezember 1886, S. 10. Vgl. Terty F. FRIEDMAN, Foggini's statue of Queen Anne, in: Klaus LANKHEIT, Kunst des Barock in der Toskana. Studien zur Kunst unter den letzten Medici, München 1976, S. 39-56.

40 Vgl. The Morning Post, 6. Juni 1888, S. 7; The Times, 16. Dezember 1886, S. 10. 
nen hatte, eine Statue für die Gattin Edwards VII. Queen Alexandra eingeweiht werden konnte. Sie wurde im Haupthof jenes London Hospital im EastEnd plaziert, als dessen Präsidentin sie gewirkt und das sie mit karitativem Engagement unterstützt hatte ${ }^{41}$. Die Denkmalhülle fiel unter den Klängen der Nationalhymne, so daß trotz Abwesenheit von Vertretern der Regierung oder des Parlaments eine dezidiert nationale Symbolik durchaus intendiert schien. Die kurzen Reden stellten darauf ab, daß Alexandra nicht nur die medizinische Ausstattung des Krankenhauses verbessert, sondern in den Jahren des Burenkrieges auch auf ihre Kosten Krankenschwestern nach Südafrika zur medizinischen Versorgung der Soldaten an der Front hatte entsenden lassen ${ }^{42}$. Im Spiegel des Monuments trat nicht nur eine ebenso patriotisch wie human motivierte Caritas der Geehrten, sondern auch das Leid militärischer wie ziviler Teile der Gesellschaft in den Blick, dessen Linderung gefördert zu haben emotional einnehmend wirkte:

The Queen had endeared herself to all English women and English men by her ready, sincere, and genuine sympathy with suffering and misery in any form. (...) We had a long line of illustrious consorts, (...) yet there was not one in that illustrious line who had been so enthroned in the heart of the nation as Her Majesty Queen Alexandra ${ }^{43}$.

Mit der symbolischen Verortung der Königin im emotionalen Innersten der »nation« war nun endgültig ein Deutungsmuster gewählt worden, das Affinitäten mit dem preußisch-deutschen Luisenmythos zeigte. Auch hier wurden quasi-hagiographische Topoi mobilisiert und schematisch feste narrative Stufen beschritten vom ersten Erscheinen Alexandras in der Zeit eines »deep sorrow of the nation « - das hier von der Trauer um den Prince Consort herrührte - zur Spende ebenso kollektiven Trostes für »court and people« in ihrer Rolle als Gattin des Prince of Wales, bis zum selbstlosen Engagement für den notleidenden Teil der militärischen und zivilen Gesellschaft, das aufgrund persönlicher Leiderfahrung der königlichen Retterin besonders authentisch wirkte ${ }^{44}$.

Die Plazierung des Alexandra-Denkmals in der Krankenhausanlage geriet unter diesen Voraussetzungen zu einer Symbolhandlung, die über die unmittelbare Motivierung dieser Ehrung mit der königlichen Präsidentschaft und Unterstützung für das London Hospital hinausreichte, indem sie Leidpotentiale und Linderungsbedarf als eine Art humane Konstante der nationalen Gesellschaft implizierte ${ }^{45}$. Gleichwohl schlugen solche Beobachtungen nicht etwa in die Forderung nach Angleichung sozialer Lebensverhältnisse um, zumal ein

${ }^{41}$ Vgl. The Daily Telegraph, 11. Juli 1908, S. 6; The Standard, 11. Juli 1908, S. 9.

42 Vgl. ibid.

${ }^{43}$ Ibid.

44 Vgl. ibid.: "Forty-five years ago she came to these shores at a time of deep sorrow for the nation. (...) She had herself known sorrow, (...), and it was fitting there should be such a memorial to her.«

45 Vgl. ibid. 
Zusammenhang mit dem verarmten Londoner Eastend als dem Sitz des Krankenhauses nicht ausdrücklich hergestellt wurde. Auch die weithin kommentarlos berichtende Presse stellte entsprechende Verbindungen nicht her. Der Daily Telegraph etwa bezeichnete das London Hospital zwar als Sammelstelle nicht nur gesundheitlichen Elends in einem »congested district ${ }^{46}$. Armut und Krankheit wurden indessen nicht in ein kausales Verhältnis gesetzt, die "poor" und "suffering" schafften eher das depravierte Betätigungsfeld und den Leidenshintergrund, vor dem sich Alexandra gleichsam als humanitäre Heldin bewähren konnte.

\section{Vergleich}

$\mathrm{Da} B$ in Paris anders als in London und Berlin zahlreiche Projekte für Frauendenkmäler scheiterten, änderte nichts an dem Umstand, daß andere, weniger politisierte Projekte in der Tat gelangen und die Pariser Denkmallandschaft mit den weiblichen République-Allegorien immerhin über eine idealisierte Frauengestalt als Staatsemblem und mit den Jeanne d'Arc-Denkmälern über eine gleich von mehreren Kultstiftern in Anspruch genommene Heroin verfügte.

Faktisch unternahmen überhaupt nur in Paris Denkmalstifter den Versuch, weibliches Personal nach gleichen Kriterien wie ihr männliches Pendant monumental zu ehren und damit dezidiert politische Konnotationen zu verbinden. Im Falle des Louise Michel-Projekts war wohl eine Kombination aus allgemeinem Ressentiment gegen die gleichberechtigte Frauenehrung und vor allem politischer Mißliebigkeit der Sozialistin, der im übrigen auch mit keiner männlichen Kultfigur in Paris durchsetzungsfähig wurde, für die Verhinderung des Unterfangens verantwortlich. War hier noch der politische Vorbehalt womöglich gleichauf mit dem geschlechtsspezifischen, zeugte spätestens das Projekt des »Monument de la Femme Française« von dem Umstand, daß Helden- und Märtyrertopoi von Frauen nicht in gleichem Maße reklamiert und in öffentlicher Anerkennung perpetuiert werden konnten, wie dies für Männer galt. Die aktive Teilhabe an der memorialen Bewältigung der Niederlage von 1871 wurde den Frauen damit verweigert. Die Vereitelung des Royer-Denkmals war demgegenüber wohl eher den Kriegswirren zuzuschreiben, in denen das langsam angelaufene, aber bereits weit gediehene Projekt nicht mehr zu Ende gebracht werden konnte. Selbst angesichts der mißlungenen Projekte nahm sich die Pariser Denkmal(projekt)landschaft damit zumindest virtuell deutlich emanzipatorischer aus, als das Symbolterrain der beiden anderen Hauptstädte, 
wo vergleichbares Personal nicht auch nur entfernt als denkmalwürdig anvisiert worden ist.

An den Berliner Monarchinnendenkmalfesten nahm der Kaiser persönlich teil und verlieh den Festen damit einmal mehr den Charakter pietätvoller monarchischer Stiftung. Während der Kaiser für die sakral geprägte Einweihung des Luisendenkmals die Familiarisierung der Nation im Zeichen ihres Kults anordnete und auf militärische Symbole und Rituselemente verzichtete, hielten in die Feier des Augusta-Denkmals 15 Jahre später zum Zeichen vorangeschrittener demonstrativer Militarisierung der Kaiseraura militärische Kultelemente mit der abschließenden Truppenparade Einzug. Die Programmatik des Denkmals für Luise lag nahe bei derjenigen, die man in Berlin bereits Ende der 1850er Jahre in Oranienburg an entlegenerer Stelle hatte beobachten können. Sakralisierung der Volksheiligen zum einen und Verbürgerlichungstopoi zum anderen markierten die Bandbreite von Bedeutungsassoziationen. $\mathrm{Zu}$ gleich wurde einmal dezidiert auf die preußische Nation nicht nur als monarchisch und christlich-religiös konnotierte Ordnung, sondern auch als Leidenserfahrungsgemeinschaft und gleichwohl zu Erhebung und machtstaatlicher Emanzipation berufenes Volk abgehoben, zum anderen und unvermittelt daneben aber auch die Nation als bürgerliche Werte- und Tugendgemeinschaft und Luise als Exempel eines weiblichen Sittenkodex imaginiert. In der öffentlichen Rezeption durch den politischen Konservativismus blieb das Luisendenkmal in der von den Stiftern intendierten Weise eklektisch, indem Luise als Affektgestalt, nicht aber als Begründerin einer hochgradig emanzipatorischen CaritasArbeit angesprochen wurde. Im Rahmen der Augusta-Feier erwiesen sich diese Deutungsmuster wiederum als gültig, zumal die Kaisergattin nicht über den Nimbus der providentiellen Preußenretterin verfügte, sondern in der Tat eher nach bürgerlichen Verhaltensnormen um ihrer Caritas willen belobigt wurde. Die linke Vision von der tatkräftig-gleichberechtigten Frauengestalt blieb demgegenüber Episode.

Ein äußerst ähnlicher Würdigungsmodus galt in London. Während die Plazierung der Anne-Statue noch ein extrem formalisierter Akt blieb, in dessen Zug die Metropole ihre monarchische Loyalität bekundete, wies die Ehrung Queen Alexandras große Affinitäten zum preußisch-deutschen Luisenkult auf. Die Alexandra-Legende mußte indessen schon allein deshalb dem LuisenMythos unterlegen bleiben, weil die englische Königin-Witwe zum Zeitpunkt der Ehrung noch lebte, so daß sich etwa quasi-religiöse Epiphanie-Visionen verbaten. Ebenso differierte die Dimension kollektiver Leiderfahrung, vor deren Hintergrund sich das erste Auftreten der säkularen Heiligen vollzog: die Kopplung der Luisenlegende an das preußische Desaster von 1806/07 nährte kompensatorische nationale Heilserwartungen und Revanchefiktionen, die der Alexandra-Figur selbst unter den Bedingungen des zwischenzeitlich unpopulär gewordenen, am Ende aber gewonnenen Burenkrieges fremd bleiben mußten. 
Das Grundmuster der weiblichen Kultfigur freilich, nach dem nicht gute Regentschaft, politisches oder gar militärisches Vermögen, sondern monarchische Abkunft, Caritas und Emotion als Leistungsmerkmale zählten, die die Figur mit der Nation affektiv ins Verhältnis setzten, waren hier wie da überdeutlich. 
